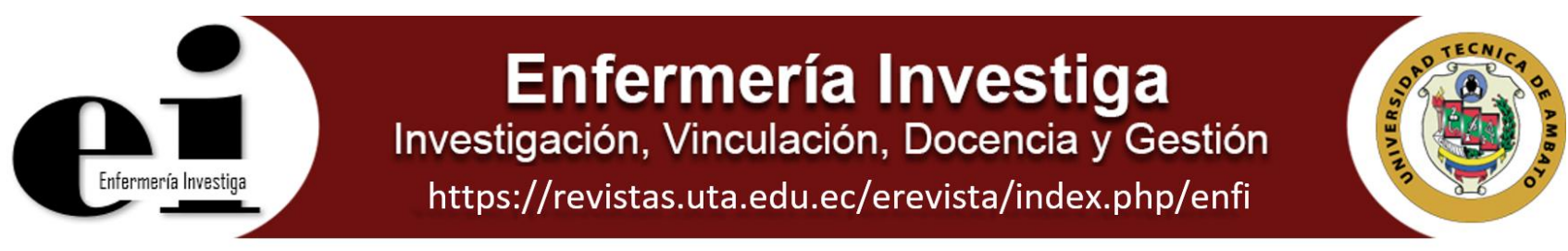

\title{
ATENCIÓN DE CALIDAD A PACIENTES CON DEFICIENCIA AUDITIVA Y VERBAL, POR PARTE DEL PERSONAL DE ENFERMERÍA DEL HOSPITAL GENERAL SANTO DOMINGO
}

\section{THE QUALITY OF ATTENTION GIVEN TO PATIENTS WITH AUDITORY AND VERBAL DEFICIENCY FROM THE PERSONNEL IN THE NURSING AREA OF THE GENERAL HOSPITAL OF SANTO DOMINGO}

Walter Patricio Castelo Rivas http://orcid.org/0000-0002-4276-087, Karla Karolina Chavarría Zambrano http://orcid.org/0000-0002-2008-5107, Carla Mishell Vaicilla Vega http://orcid.org/0000-0002-1766-671X, Joselyn Brigitte Vanegas Viñan http://orcid.org/0000-0002-9773-9236

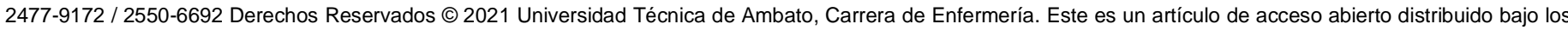

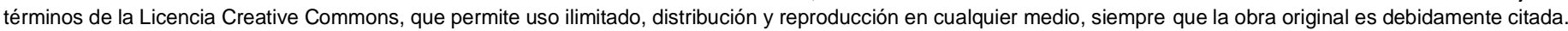

Recibido: 01 de septiembre 2021

Aceptado: 28 de noviembre 2021

\section{RESUMEN}

Introducción: La atención que se brinda a los pacientes sordomudos depende de la relación y comunicación entre el personal de enfermería y los usuarios, pero a pesar de que se trata de una población en ascenso progresivo, existe un déficit de formación, por lo que la atención lo convierte en un desafío para estos. profesionales. Objetivo: Analizar la calidad de atención brindada a las personas sordomudas, en base al desempeño de los profesionales de Enfermería. Métodos: Se utilizó un enfoque cualitativo y diseño fenomenológico. La población escogida fue de 12 enfermeros/as, 9 que laboraban en el área de emergencias y 3 en consulta externa del Hospital General Santo Domingo, utilizando el tipo de muestreo por conveniencia se seleccionó a una muestra de 8 profesionales, se utilizó entrevistas semiestructuradas, las cuales permitieron ahondar en la percepción de los enfermeros/as ante el tema. El análisis de datos se realizó mediante la aplicación Atlas ti Versión 9. Resultados: Se evidenció que, en la atención a personas sordomudas, la comunicación enfermero-paciente tiene muchas falencias, las cuales complican el acceso a la salud de esta población debido a que no se logra comprender completamente las necesidades que mantienen estos usuarios, o a su vez se necesita de terceros para llegar al individuo. Además, existe desinterés de los enfermeros/as, universidades e instituciones de salud en cuanto a la formación integral profesional. Conclusiones: La atención de calidad se ve influenciada negativamente debido a la complejidad de la comunicación entre el enfermero y el paciente sordomudo.

Palabras claves: personal de enfermería; pérdida auditiva; trastornos del desarrollo del lenguaje; comunicación; calidad de la atención de salud.

\section{ABSTRACT}

Introduction: The care provided to deaf-mute patients depends on the relationship and communication between the nursing staff and the users, but despite the fact that it is a population in progressive ascent, there is a training deficit, so the care is makes it a challenge for them. professionals. Objective: To analyze the quality of attention given to deaf-mutes, based on the performance of the professionals in Nursing. Methods: A qualitative focus and phenomenological design were used. The population chosen was of 12 male/female nurses, nine of which worked in the emergency area and three in the outpatient area of the General Hospital of Santo Domingo, using a type of convenience sampling, a sampling of eight professionals was selected, semi-structured interviews were used, of which allowed for a deepening of the perception of male/female nurses on this subject. The data analysis was done with the support of the application Atlas ti Version 9. Results: It was evidenced that, in the attention given to deaf-mute people, the communication nurse-patient has many flaws, those of which complicate access to health on the part of this population due to the fact that the needs of these service users cannot be fully understood. At the same time, third parties are needed in order to reach the individual. Besides, there exists a lack of interest on the part of the male/female nurses, universities, and health institutions regarding the integral professional performance. Conclusions: Quality attention is seen to be negatively influenced due to the complexity of the communication between the nurse and the deaf-mute patient.

Keywords: nursing personnel; auditory loss; language development disorders; communication; quality in the attention to health. 


\section{INTRODUCCIÓN}

El rol del personal de enfermería se basa en brindar atención integral a todas las personas; para lograr este objetivo, es necesario mantener un diálogo efectivo entre el equipo de enfermería y el usuario, por estas razones se considera a los enfermeros/as como proporcionadores de cuidados en los establecimientos de salud (1), los mismos que, deben estar preparados para asistir a diversos pacientes, entre ellos la población sordomuda, que desde su nacimiento padecen de sordera y como consecuencia mantienen pérdida o trastornos del habla (2).

Como se ha mencionado anteriormente, para el desarrollo del proceso asistencial es fundamental la comunicación, la cual se refiere a la transferencia de información entre personas, logrando el análisis y el entendimiento de las ideas. La interacción con los pacientes sordomudos se ha convertido en un verdadero reto para el equipo sanitario y la red nacional de salud, interfiriendo en el desarrollo de la atención de calidad, definida como el grado en que los cuidados aplicados alcanzan los máximos estándares deseados (1).

De acuerdo con el estudio ejecutado por Francisqueti et al., la comunicación es un instrumento primordial que permite al enfermero obtener y comprender la información proporcionada por las personas con pérdida de audición (3). A su vez, una investigación realizada en Brasil, indica que los profesionales de enfermería hacen grandes esfuerzos para lograr comunicarse y brindar cuidados a los pacientes sordomudos, mediante estrategias como: el lenguaje escrito y corporal (gestos y mímica), lenguaje de señas, lectura de labios, ilustraciones, dactilología, traductor o intérprete y por medio de la persona que acompaña al paciente, que se ha convertido en el principal intermediario de la comunicación asistencial (4). Además, Martínez y Pinos afirman que los trabajadores de la salud consideran importante contar con conocimientos suficientes para comunicarse con pacientes que padecen esta deficiencia y así satisfacer sus necesidades (5). Por otro lado, en Chile se analizó la formación de los profesionales de salud, la cual debe ser en base a contextos de diversidad en pacientes, especialmente aquellos con deficiencia auditiva y verbal (6).

Según la Organización de las Naciones Unidas la población sordomuda se ha incrementado, actualmente existen 72.000.000 de personas con esta discapacidad en el mundo; el $80 \%$ habita en países subdesarrollados y emplean más de 300 diversas lenguas de señas (7); también, estos individuos son susceptibles a sufrir carencias en los servicios de atención, específicamente en el acceso a la salud, con la afectación de su bienestar biopsicosocial. (8). Asimismo, en una publicación ejecutada por Human Rights Watch, se evidenció que la dificultad para comunicarse influye negativamente en el derecho a la salud, debido a la escasez de formatos para esta comunidad; de igual manera, se viola el derecho de la privacidad al apoyarse de terceras personas (9).

En América Latina se encuentran aproximadamente 36.000.000 de personas que presentan dificultad auditiva y verbal (10), de acuerdo a un estudio realizado en New York, existe falencia en el aprendizaje del lenguaje de señas por parte del mismo grupo afectado, lo que complica su comunicación y por lo tanto, la atención a sus necesidades (9). Es por esto que, el $66 \%$ de los pacientes no comprenden las indicaciones dadas por los enfermeros/as, incluido el diagnóstico y tratamiento, causando en estos individuos sentimientos de inseguridad, ira, impotencia y desilusión al no saber cómo comunicarse o entender al personal de enfermería; hay que mencionar que, la falta de medios asequibles de información para estas minorías impiden la inclusión a los eventos de fomentación de la salud, lo que conlleva a que sean personas susceptibles a enfermedades que pueden ser evitadas (4).

Por otra parte, en Ecuador, según el Registro Nacional de Discapacidad existía en el año 201550.838 individuos con discapacidad auditiva (11), actualmente, esta cifra se ha incrementado a 66.558 (12). En Santo Domingo una ciudad ecuatoriana, la cantidad registrada muestra 1.486 individuos sordos (11). Por lo tanto, se evidencia un crecimiento significativo de esta población.

El presente estudio se enfoca en responder las siguientes interrogantes:

¿Qué cuidado otorgan los profesionales de Enfermería de las áreas de emergencias y consulta externa para brindar atención a pacientes sordomudos?

¿Cómo se lleva a cabo la comunicación enfermero-paciente durante la atención a personas sordomudas?

¿Cuál es la formación que tienen los profesionales de enfermería para cubrir con las necesidades asistenciales y comunicativas de los pacientes con deficiencia auditiva y verbal?

Además, es necesario destacar que a pesar que existe una gran cantidad de personas con deficiencia auditiva y verbal, se evidencia el desinterés de los enfermeros/as en relación a la comunicación con pacientes sordomudos. Se debe agregar que, existe escasa investigación acerca del tema en Santo Domingo de los Tsáchilas. Por esta razón, es de suma importancia realizar el presente estudio para analizar la calidad de atención brindada a las personas sordomudas, en base al desempeño de los profesionales de Enfermería.

\section{MÉTODOS}

Investigación con enfoque cualitativo y un diseño fenomenológico, debido a que se buscó analizar las experiencias del personal de enfermería en relación a la atención brindada a pacientes con deficiencia auditiva y verbal y de esta forma conocer cómo subjetivamente perciben su realidad (13). Se seleccionó por medio de un muestreo por conveniencia a 12 enfermeros/as: 9 del área de emergencias y 3 de consulta externa del Hospital General Santo Domingo, (14); adicionalmente, se aplicaron criterios de inclusión: tener el título de licenciatura en Enfermería y experiencias en la atención de pacientes sordomudos. En cuanto a los criterios de exclusión: estudiantes de enfermería, enfermeras auxiliares y la negativa de participar en la investigación.

El material empleado para la recolección de datos fueron entrevistas semiestructuradas; con preguntas abiertas en un cuestionario previamente validado por expertos, las mismas que fueron grabadas y posteriormente transcritas, con ello se conoció la experiencia de los enfermeros en relación a su percepción del cuidado aplicado al paciente sordomudo. En cuanto al equipo para la grabación, se utilizó un dispositivo móvil Samsung Galaxy J7 Prime con Voice Recorder versión 21.0.22.166.

Para analizar los datos se empleó el software Atlas ti versión 9, en el cual se ingresaron las entrevistas realizadas a los 
participantes, organizadas en un solo documento Word, luego se procedió a subrayar las coincidencias encontradas en las preguntas de cada una de las intervenciones, de manera que se establecieron los códigos y a la vez se vincularon aquellos que guardaban relación entre sí. En consecuencia, se crearon redes que representaron las categorías inductivas, para su adecuada identificación se les asignaron un color, estas fueron: relación y comunicación con el paciente sordomudo, color verde; estrategias para lograr la comunicación con el paciente sordomudo, color morado; satisfacción del paciente sordomudo ante la atención brindada, color rosado y formación de los profesionales para brindar atención a pacientes sordomudos, color azul. Con ello, se relacionó la información obtenida y se sintetizó las ideas para construir el informe final de resultados.

Este estudio mantuvo la confidencialidad en cuanto a los datos personales de cada uno de los enfermeros/as involucrados en la investigación, se utilizó colores elegidos por los mismos participantes como sus pseudónimos, además la entrevista fue realizada previa autorización y consentimiento informado de cada uno de los profesionales. Se debe agregar que, se consiguió la aprobación y autorización de las autoridades pertinentes para la realización del estudio. Asimismo, se cuidaron los principios éticos de respeto, autonomía y no maleficencia.

Para tener acceso a los participantes del estudio, se realizó un contacto inicial de las investigadoras con la máxima autoridad de la institución de salud mediante un oficio, posteriormente, se tuvo un conversatorio con los encargados del departamento de investigación y director de enfermería; se accedió a las respectivas áreas por medio de las coordinadoras, manteniendo un primer acercamiento con la población donde se socializó el tema a tratar y el consentimiento informado, por último, se estableció un cronograma para las entrevistas de acuerdo a la disponibilidad de cada licenciado/a.

\section{RESULTADOS}

En base al análisis desarrollado, se logró categorizar la información obtenida en las entrevistas realizadas al personal de enfermería, con la ayuda del software, determinando lo siguiente:

\section{Categoría 1. Relación y comunicación con el paciente sordomudo}

En cuanto a la relación y comunicación que mantienen los profesionales de enfermería con pacientes sordomudos, se pudo determinar que existe dificultad en el desarrollo de la atención a estos usuarios. Un profesional entrevistado menciona que:

\begin{abstract}
"Se considera un poco complicado debido a que no son pacientes que tratamos a diario y ellos utilizan otro lenguaje como señas" (Verde).
\end{abstract}

Normalmente, el proceso asistencial que se maneja en la atención sanitaria, se desarrolla mediante un lenguaje verbal, lo que impide la comunicación cuando se trata de un paciente que no utiliza este tipo de lengua. Asimismo, otra enfermera señala:

"Hay casos en que ni los mismos pacientes sordomudos entienden el lenguaje de señas. Eso es un problema tanto para ellos como para nosotros" (Morado).
Cabe destacar la importancia del conocimiento de la lengua de signos, tanto del profesional como del paciente para conseguir el entendimiento y brindar cuidados adecuados. Por otra parte, se ve afectada la autonomía del paciente debido a que, en algunos casos, no se le está informando personalmente, sino que se emplea una comunicación mediada a través de un familiar. Así, una de las entrevistadas comenta su experiencia ante la relación con sordomudos:

"Uno no llega directamente al paciente. Por lo general los enfermeros nos comunicamos con el familiar" (Rosado).

Es así que, muchas veces no se logra saber todas las necesidades que tiene el usuario o no se toma en cuenta su decisión ante los procedimientos a realizar, sino que se opta por identificar la información dada por el acompañante. Además, aspectos como la falta de protocolos, escasa capacitación de la atención de pacientes sordomudos durante formación universitaria, poca experiencia y la incomprensión del individuo ante los cuidados que se proporcionan, impiden que se brinde una atención de calidad y una relación enfermero-paciente efectiva.

\section{Categoría 2. Estrategias para lograr la comunicación con el paciente sordomudo}

Entender a otra persona es una tarea compleja, más aún cuando esta padece de una capacidad diferente que lo aísla de la comunicación verbal, lo cual se vuelve un reto para los profesionales de enfermería al momento de brindar cuidados a estos pacientes. Esta situación impulsa a los enfermeros a buscar estrategias comunicativas favorables para actuar ante estos casos, entre ellas: lectura de labios, mímicas, gestos, vocalizar, leer, escritura, señalética, demostración mediante objetos y lenguaje de señas. Una de las entrevistadas manifiesta que:

"Estas personas desarrollan otras habilidades como puede ser la lectura de los labios. Entonces procuraré hablarle mirándole a la cara y vocalizando bien, o con el apoyo del familiar o cuidador. Muchas veces pues a lo mejor le enseñas una jeringuilla, le dices te voy a pinchar y estamos pendiente de observar sus gestos" (Rosado).

Para los profesionales de la salud nada es imposible cuando se trata de cuidar a otro, buscan la forma de hacerse comprender y brindar cuidados de calidad. Otra enfermera expresa:

"Pregunto si sabe lenguaje de señas, si sabe utilizó señas, y si hay pacientes sordomudos que sí saben escribir, entonces que se exprese en forma escrita" (Azul).

Una minoría de la población sordomuda emplea el lenguaje de señas, mientras que otros no tienen acceso a la educación apropiada para aprenderlo, por lo que se les dificulta la comunicación (15). Otra profesional de enfermería recalca la forma en que se comunica con estos individuos:

"Lo único que he podido hacer hasta ahora es comunicarme a través del familiar (...) Hasta el momento es lo más apropiado para mí, hacerlo a través del familiar porque ellos son los que conocen al paciente y saben las maneras de 
cómo hacerlo quizás entender o tratarlo y ellos nos ayudan igual a nosotros" (Rosado).

Es así que, de acuerdo con el conocimiento teórico y la experiencia de los profesionales entrevistados, la estrategia de comunicación más utilizada es el apoyo de un familiar, debido a que facilita el entendimiento, la relación del usuario y el enfermero/a.

\section{Categoría 3. Satisfacción del paciente sordomudo ante la atención brindada}

La manera de expresarse de las personas con deficiencia auditiva y verbal orientan al profesional de enfermería, para saber si se logró la adecuada comprensión de su proceso de atención, algunas de las expresiones positivas evidenciadas por el equipo de enfermería son: sonrisas, grado de relajación, tranquilidad, subir y bajar la cabeza haciendo intención de afirmar que se entendió y que está satisfecho de los cuidados proporcionados; por el contrario, en representación negativa, se encuentra el estado de nerviosismo. De acuerdo a su experiencia, una de las entrevistadas menciona:

\begin{abstract}
"Uno tal vez lo puede evidenciar viendo la tranquilidad del paciente al momento, por ejemplo, si le voy aplicar una medicación y el familiar le hace comprender que es lo que se le va a hacer, hay unos como que sí están tranquilos, pero hay otros que no, como que no entienden o no se sienten seguros" (Rosado)
\end{abstract}

Por otro lado, una enfermera involucrada en el estudio manifiesta que una forma básica de medir el nivel de comprensión del paciente es:

"Tratando de ver que haga un signo de afirmación, que sería subir y bajar la cabeza, en caso que él diga que no, pues nos haría lo contrario" (Azul)

\section{Categoría 4. Formación de los profesionales para brindar atención a pacientes sordomudos}

Es importante recalcar que, la formación de los profesionales influye en la atención de calidad de las personas sordomudas. Por ello, los profesionales, universidades e instituciones de salud deben preocuparse y priorizar la atención a los grupos minoritarios, entre ellos los pacientes sordomudos, debido a que se ve obstaculizado el acceso a la atención sanitaria de estos usuarios, por no lograr una comunicación efectiva, la cual pone en riesgo el bienestar de estos pacientes y sus derechos se ven vulnerados.

Con respecto al desinterés del profesional, se puede ver influenciado debido a la falta de capacitaciones de acuerdo a la temática, a su vez la accesibilidad a los mismos, déficit de autoformación, falta de entrenamiento y actualización continua. Una de las entrevistadas menciona que:

"Cada uno por su parte debería preocuparse por la atención a este tipo de pacientes por medio de la autoformación" (Rosado).

Por otra parte, el desinterés de las universidades se ve reflejado en la falta de una asignatura que abarque los cuidados a pacientes con capacidades especiales, como aquellos con deficiencia auditiva y verbal. Por lo tanto, los profesionales adquieren experiencia fuera de la formación de pregrado. Uno de los involucrados afirma:

\section{"No existe preparación dentro de la Universidad para el proceso de atención de pacientes sordomudos, porque literalmente no se explica, es decir, es nula nuestra preparación. Entonces la experiencia la tengo que obtener después de graduada" (Verde).}

Asimismo, las instituciones de salud muestran desinterés ante esta situación debido a la carencia de capacitaciones a sus trabajadores y también, la escasa implementación de herramientas que contribuyan al mejor desempeño de los profesionales, en todas las áreas de atención. Una de las enfermeras entrevistadas recalca:

"Este es un tema bastante amplio y que pocas personas o pocas instituciones han ido por ese camino se puede decir. De hecho, aquí en el hospital, no se habla mucho o no se ha abordado mucho el tema" (Morado).

Cabe recalcar que, algunas instituciones externas se interesan por el personal de enfermería en cuanto a su formación, mediante la gestión de capacitaciones para brindarles estrategias de comunicación y así facilitar el proceso asistencial, que favorezca a la población sordomuda y se logre una atención de calidad. Una de las enfermeras menciona:

"El personal del Centro Fe y alegría nos explicó el lenguaje de mímicas y nos enseñaron cómo debemos saludar" (Morado).

\section{DISCUSIÓN}

En cuanto a los hallazgos en la investigación, se logró apreciar que la comunicación entre la población sordomuda y los profesionales de enfermería es compleja y dificulta el desarrollo de la atención. Estos resultados guardan relación con lo que sostienen Martínez y Cuenca, al afirmar que los enfermeros/as se sienten impotentes ante la incomprensión de la información dada al paciente al momento de brindar sus cuidados $(5,16)$.

Por otro lado, la formación juega un rol fundamental en el proceso asistencial de una persona con deficiencia auditiva y verbal, debido a que al existir una barrera comunicacional entre las dos partes puede generar malos entendidos, que conlleven a tergiversar los datos proporcionados y cometer acciones erróneas en su plan de cuidados, poniendo así su vida en riesgo. En concordancia, Francisqueti et al. mencionan que el déficit de preparación profesional para este grupo minoritario influye de manera negativa en la adecuada atención de los mismos $(3,17,18)$.

En lo que respecta a los métodos más aplicados por el enfermero/a para llegar al paciente se considera el apoyo de un familiar, datos similares se expresan en el artículo de Santana y Freire, el cual refiere que la primera opción de una persona sordomuda para asistir al sistema sanitario es hacerlo con un intermediario, como puede ser un familiar, amigo o conocido $(4,19,20)$. Se debe agregar que, en cuanto al uso del lenguaje de señas en la comunicación, es relevante su aprendizaje por parte del equipo de enfermería y el individuo para lograr una atención de calidad y calidez. Como se enfatiza en el estudio de 
Francisqueti et al., uno de los impedimentos más comunes para relacionarse con este tipo de usuarios es el desconocimiento de la lengua de signos $(3,21)$.

El análisis también determinó que en muchos casos los profesionales de enfermería se enfrentan a la incertidumbre de no saber con certeza si sus pacientes comprenden las indicaciones y cuidados prestados. Ante esto, solo se pueden guiar mediante gestos básicos que demuestren afirmación o negación. Afirmando esta idea, Matus et al. describe como frustrante a las sensaciones por parte de los enfermeros/as al no comprender las necesidades del usuario al que presta sus servicios $(6,22)$.

Por último, existe poco interés de las universidades por reestructurar la malla académica con una asignatura independiente, que desarrolle las estrategias de comunicación y los cuidados esenciales para esta población. Diversos autores como Francisqueti et al., Lopera et al., Hualde y Bornholdt et al., en relación a esta idea y con respecto a la experiencia, los profesionales de enfermería refieren no estar aptos para brindar una atención oportuna y calificada a este grupo vulnerable, aunque algunos una asignatura optativa en su plan de estudios $(3,23,24,25)$.

Al momento de realizar el estudio se percibió algunos obstáculos que dificultaron recabar la información de los profesionales de enfermería. Esto debido a los horarios y a la demandante carga laboral, que impedían la realización de las entrevistas en el día y hora programada.

\section{CONCLUSIONES}

La atención otorgada por parte de los profesionales difiere en dos aspectos, por una parte, los profesionales creen otorgar un buen servicio desde el punto de vista del proceso asistencial, pero la interacción directa con los pacientes no es la adecuada. Por lo tanto, la comunicación que existe entre los profesionales de enfermería y los individuos sordomudos es compleja. El factor más importante que influye en la interacción con este tipo de usuarios es la falta de conocimientos. Además, una de las formas más utilizadas por los profesionales para comunicarse con esta población es a través de un familiar, resaltando que existe incertidumbre por parte de los enfermeros al momento de atender a estas personas, por tanto, es necesario que las instituciones educativas complementen el déficit de conocimiento con asignaturas dentro del pensum académico acerca del cuidado y manejo efectivo para este grupo vulnerable.

CONFLICTO DE INTERESES: Ninguno expuesto por los autores

FINANCIAMIENTO: No hubo fuentes de financiamiento.

\section{REFERENCIAS}

1. Descriptores en Ciencia de la Salud: DeCS [base de datos en Internet]. Sao Paulo: BIREME / OPS / OMS; 2021. Disponible en: https://decs.bvsalud.org/es/

2. Real Academia Española. [base de datos en Internet]. Madrid: RAE;. Disponible en: https://dle.rae.es/sordomudo

3. Francisqueti V, Gonzalez L, Costa H, Ferraz E, Dos Santos G, Ramos M. Formação de profissionais para a atenção a saúde de deficientes auditivos. Rev. Enferm. UFSM, 2018; 8(4): 649-60. doi: 10.5902/2179769230966

4. Santana Santos A, Freire Portes A. Percepciones de sujetos sordos sobre la comunicación en la Atención Básica a la Salud. Rev.Lat.-Am.Enferm, 2019;27:1-9. Disponible en: https://cutt.ly/6WyEWTB

5. Martínez E, Pinos J. Comunicación del enfermero/a con pacientes que padecen deficiencia auditiva y verbal en el Hospital General Santo Domingo [tesis]. Santo Domingo-Ecuador: Pontificia Universidad Católica del Ecuador sede Santo Domingo; 2019 [citado 2021 jun. 29]. Disponible en: https://cutt.ly/SWyEOFv

6. Matus O, Ortega J, Pérez C, McColl P, Navarro N, Silva V, et al. Formación clínica en carreras de la salud: ¿dónde se incluye la diversidad? Rev. méd. Chile. 2020; 148(4): 444-51. Disponible en: https://cutt.ly/vnHKpUT

7. Organización de las Naciones Unidas. Día Internacional de las Lenguas de Señas, 23 de septiembre [base de datos en Internet]. Nueva York: 2021. Disponible en: https://cutt.ly/smoMKnX

8. Organización Mundial de la Salud. Discapacidad y salud [base de datos en Internet]. Ginebra: 202. Disponible en: https://cutt.ly/mmo1Zds

9. Human Rights Watch. El lenguaje de señas, un componente clave para los derechos de las personas sordas [base de datos en Internet]. Nueva York: HRW. Disponible en: https://cutt.ly/dWyEZJV

10. Meléndez J, Hernández L, Rosas K, Chacón T, Ríos A. ¿Cómo está el acceso a la educación para las personas sordas en América Latina? [base de datos en Internet]. México: Distintas Latitudes; 2018. Disponible en: https://cutt.ly/SWyEBSH

11. Consejo Nacional para la Igualdad de Discapacidades. Total de personas con discapacidad registradas en el registro nacional de discapacidad [base de datos en Internet]. Quito: CONADIS; 2021. Disponible en: https://cutt.ly/dWyE8d1

12. Ministerio de Salud Pública. Registro Nacional de Discapacidades [base de datos en Internet]. Ecuador: 2015. Disponible en: https://cutt.ly/QWyE6wH 
13. Hernández R, Fernández C, Baptista P. Metodología de la investigación. 6th. ed. México: McGraw-Hill; 2014. Disponible en: https://cutt.ly/jWyRPO3

14. Otzen T, Manterola C. Técnicas de Muestreo sobre una Población a Estudio. Int. J. Morphol. 2017; 35(1): 227-32. Disponible en: https://cutt.ly/lmAtqWO

15. Telesurtv.net. [programa de televisión]. Caracas: ajb-JGN; 2020. Disponible en: https://www.telesurtv.net/news/dia-internacionallenguas-senas-datos-curiosos-20190919-0040.html

16. Cuenca Arteaga M, Illapa Sagñay R. Intervenciones de enfermería en pacientes pediátricos con discapacidad auditiva [tesis]. Chimporazo-Ecuador: Universidad Nacional de Chimborazo; 2019. Disponible en: https://cutt.ly/nT5bbPx

17. Fernández I, López L, Guillamón L. Estrategias de comunicación en la interacción terapéutica en usuarios de edad avanzada en circunstancias de sordera. Ágora de Salut, 2019; 6: 99-08. Disponible en: http://repositori.uji.es/xmlui/bitstream/handle/10234/185577/11_Ferna\%cc\%81ndez\%2c_Lo\%cc\%81 pez.pdf?sequence=1\&isAllowed $=\mathrm{y}$

18. Madero K, Vallejo S, Castro V. Necesidades sentidas de persona con capacidades disímiles ante la Atención de profesionales de la salud. IPSA Scientia rev. cient. Multidiscip, 2021;(6):10-21. doi: 10.25214/27114406.1004

19. Cóceres A, Rovira M, Vallejos S, Auchter, M. La comunicación entre el enfermero asistencial y los pacientes con discapacidad auditiva en un hospital público de la ciudad de Corrientes. Año 2020. Rev. Fac. Med. Univ. Nac. Nordeste, 2021;41(1):4-16. Disponible en: http://revista.med.unne.edu.ar/index.php/med/article/download/139/127

20. Aragón A. Habilidades y métodos comunicativos del personal de Enfermería durante el cuidado de personas con limitación auditiva [tesis]. Medellín-Colombia: Corporación Universitaria Adventista; 2017. Disponible en: http://repository.unac.edu.co/bitstream/handle/11254/89/Trabajo\%20de\%20grado.pdf?sequence=1\&isAllowed=y

21. Sánchez B. Experiencias de la atención enfermera a mujeres sordas en el periodo reproductivo [tesis]. Madrid-España: Universidad Autónoma de Madrid; 2019. Disponible en: https://cutt.ly/5T5KEt5

22. Barranco Morente S, Ventura Puertos P, Coronado Carvajal P. Estrategias de comunicación de los profesionales de enfermería con personas sordas o ciegas. Index Enferm, 2016; 25(4): 253-57. Disponible en: http://scielo.isciii.es/scielo.php?script=sci_arttext\&pid=S1132-12962016000300007\&lng=es.

23. Lopera Arango D, Ramírez Salazar E, Jiménez Vanegas A, Gómez Cardona J, Restrepo Castaño M. Percepción del personal de salud sobre la atención a la población sorda en el Hospital San Juan de Dios de Marinilla. Rev. Univ. Catol. Oriente, 2019; 30(44): 24-44. Disponible en: https://cutt.ly/VnHIOhE

24. Hualde Lorenzo. Programa de Formación dirigido a los alumnos de 4ํ de Enfermería de la Universidad de Zaragoza: Estrategias para mejorar la comunicación con el paciente sordo hospitalizado [tesis]. Zaragoza-España: Universidad de Zaragoza; 2019. Disponible en: https://cutt.ly/AT5bTHi

25. Bornholdt L, Pauli E, Hildebrandt L, Kinalski S, Van Der Sand I, Leite M. Cuidados de enfermagem a indivíduos com surdez e/ou mudez em instituição hospitalar. Revista Enfermagem Atual In Derme, 2019; (89): 3-7. Disponible en: http://www.revistaenfermagematual.com.br/index.php/revista/article/view/422/513 